GUEST EDITORIAL

\title{
Medical genetics, genomics and the future of medicine
}

\begin{abstract}
That the fundamental aspects of heredity should have turned out to be so extraordinarily simple supports us in the hope that nature may, after all, be entirely approachable. Her much-advertised inscrutability has once more been found to be an illusion due to our ignorance. This is encouraging, for, if the world in which we live were as complicated as some of our friends would have us believe, we might well despair that biology could ever become an exact science. (Thomas Hunt Morgan, 1856 - 1945 ${ }^{[1]}$ )
\end{abstract}

Since antiquity, the hereditary nature of health and disease has been appreciated. For instance, recognition that epilepsy, the 'sacred disease', was not truly sacred, but may have its origins in heredity, was described in Hippocratic times. Unravelling these heritable factors has, however, proven to be an ongoing challenge. For most medical conditions, the phenotype we see is the endpoint of multiple genetic and environmental factors; this includes many of the conditions seen every day in a general practice, such as type 2 diabetes, hypertension, asthma and most cancers. These multifactorial or 'complex' disorders cluster in families who share a genetic background but do not obey the rules of Mendelian inheritance. Individually, each genetic variant (GV) typically makes a small contribution and cannot be used diagnostically, but added to each other can powerfully affect the overall phenotype.

Studying the function of the gene product and interactions with other genetic modifiers and the environment can lead to new ways of thinking about therapeutic targets and interventions. Identification of both genetic and environmental factors in common conditions that are major contributors to the burden of disease is leading the way to more accurate risk stratification that can influence the implementation of appropriate health services.

New genetic and genomic technologies that have emerged since the turn of the century, alongside the exponential growth in information technology and data handling, have driven the discovery of genes, genetic variation and genetic association. This, in turn, has resulted in much greater insight into physiology and function and what can and does go wrong in biology. Until recently, most of the attention was focused on the exome - the protein-coding regions of our genome that make up only $1-2 \%$ of our DNA. However, the reduction in sequencing cost and improvements in handling big data have recently redirected much of the focus to the remainder of the genome and on how our DNA is controlled. What continues to surprise is how different we are, and this has compounded some of the difficulties in interpretation of DNA GVs that vary from the 'reference' genome - a work in progress, which is constantly updated and maintained through international collaboration. A particular challenge for Africa, where mankind first evolved, is that genetic variation is greater in African populations, while reference datasets are fewer. Clearly, Africa needs its own genome revolution.

So where to from here? On the one hand, medical genetics has long been perceived to be the study of 'rare' or unusual disorders or diseases (collectively, of course, not rare at all). On the other hand, genomic medicine means that every healthcare professional will need a working knowledge of the genome and some understanding of its complexity. There is so much known, but also so much to learn, that caution should still be exercised in interpreting genetic findings out of context, as nothing in our DNA acts in isolation. Genomic information is accumulating at a rapid rate; therefore, what we knew yesterday may be very different to what we know tomorrow. Rapid advances, coupled with easy access to information on the internet, have created expectation and anxiety in healthcare professionals and the public alike. Direct-to-consumer DNA-based testing is freely available and marketed for indications as wide ranging as cancer predisposition testing, sex determination of an embryo and personality or sporting ability associations. No wonder our patients see their genome as a crystal ball.

New findings undoubtedly drive us closer and closer to the goal of precision medicine, but the clinician will continue to play an essential role in making sense of the knowledge generated by the new 'omics'. More knowledge means more power to control our health and our environment, but brings new ethical dilemmas that need to be considered while balancing the needs of the collective and the individual.

Since the Hippocratic era, the story of medicine has been one of ever more sophisticated phenotyping: these are the symptoms of diabetes; these are the signs of diabetes; this is how diabetes affects the skin, the brain, the eyes, the kidney and the heart; these are the molecular mechanisms that underlie diabetic retinopathy, diabetic nephropathy and diabetic cardiomyopathy. Modern genetics now has a historic opportunity to complete the symmetry of this equation by bringing parallel genotyping to the traditionally phenotypic endeavour of clinical medicine. ${ }^{[2]}$

Biology and clinical medicine are amid a remarkable revolution technical advances in DNA sequencing have given us access to the molecular code governing everyone's unique phenotype, including disease susceptibility and drug sensitivity. The compelling promise of truly personalised medicine spurred the quest for methods to achieve precision genomic manipulation. In an interesting twist of scientific fate, basic research on an adaptive immune system in bacteria led to the breakthrough genomic engineering technology known as clustered regularly interspaced short palindromic repeats (CRISPR)-associated protein 9 (Cas9) (CRISPR-Cas9). Experiments focused on understanding how bacteria acquire resistance to viruses, using genomic loci known as CRISPR, uncovered the activity of the CRISPR-associated RNA-guided DNA-cleaving enzyme, Cas9. Cas9 generates double-stranded DNA breaks at sites matching the sequence of a guide RNA molecule that was designed in the laboratory to include features necessary for both Cas9 binding and DNA recognition. ${ }^{[3]}$ These new technologies have made the possibility of genome editing in humans a reality.

In the context of rapid developments in medical genetics and genomics, what does the future of medicine look like?

Precision medicine (PM). PM is an approach that proposes the customisation of healthcare, with medical diagnostics, decisions, therapies, practices and products tailored to the individual patient. Diagnostic testing employs molecular genetics, molecular and cellular biology techniques and multimodal imaging and analytics. The underlying principle of PM is not new, e.g. for more than a century, transfusion patients have been matched with donors according to blood type. Advances in genetics and genomics and growing availability of health data, make PM a clinical reality for many. Increasingly, artificial intelligence, the ability to handle big data and bioinformatics are providing a paradigm shift towards PM. 
Genome engineering and editing. With the advent of precision genome editing, the ability to modify living organisms has proceeded with remarkable speed and breadth. Any application of this technology to the human germline must be tightly coupled to deliberate ethical consideration of the consequences, both scientific and social, of introducing heritable alterations to the human population. Recently, the US National Academy of Sciences and National Academy of Medicine released their report on recommendations for human genome editing. ${ }^{[4]}$ The capacity to edit genes has existed for decades, and genetic modification is common practice in the laboratory. The heightened concern is that recent technological advances have enabled the precise editing of genomes at unprecedented speed and scale. Zinc-finger nucleases, transcription activator-like effector nucleases (TALENs) and, now, the CRISPR-Cas9 system have revolutionised scientific discovery, which has direct implications for human disease.

Genetic variants of unknown significance (GVUS). It is likely that, with increasing human mutational analysis and genotyping, there will be an exponential increase in the number of GVUS. These will add increasing complexity in interpretation and explanation to patients and families. This scenario is likely to be exaggerated in African populations, for whom comprehensive reference genetic data sets do not currently exist.

High-throughput sequencing applied to the clinic. Modern sequencing approaches have resulted in the rapid disappearance of the barriers to mutation identification in genetically heterogeneous disorders. Soon the ability to rapidly sequence all the genes known to be involved in disorders such as cardiomyopathy, long QT syndrome and retinitis pigmentosa will be at hand.

Pharmacogenomics. Even before clinical outcome data are available, the US Food and Drug Administration (FDA) has realised the importance of pharmacogenomics in clinical practice and has advised on genotypic guidance in warfarin dosing, a drug that is prescribed more than 100 million times annually globally. Currently few doctors know how to apply pharmacogenomic data in clinical practice.

Complex databases to collect genotype/phenotype information. Aided by modern bioinformatic approaches, the acquisition and interpretation of such information for patient care will become clearer for geneticists and clinicians.

Ethical, legal and social issues (ELSI). ELSI are likely to become much more prominent in academic and public discourse due to the increasing importance of genetic and genomic information for many aspects of human health and society.

Genetic counsellors. The important role that genetic counsellors must play in educating patients and the lay public regarding aspects of genetic medicine will become increasingly obvious. Doctors will not be able to accomplish the integration of genetics into medicine on their own.

Human geneticists. It is likely that many more clinics will have a multidisciplinary nature, with human geneticists playing a much more collaborative role in partnership with doctors from different disciplines.

This issue of CME, second in the series on genetics in medicine, contains 3 articles that reflect some of the complexity of genomic medicine and what the future of medicine may look like. Laing et al. ${ }^{[5]}$ review the genetic contributions to the global increasing burden of cardiovascular disease, including both monogenic and more common multifactorial disorders. Ramesar ${ }^{[6]}$ provides an introduction to epigenetics - how the genome is controlled and influenced by the environment to maintain the balance between stability and adaptability that is central to our ability to function. Finally, De Vries and Munung ${ }^{[7]}$ remind us of the ethical challenges to consider as our deeply personal genetic information gets unpacked and shared, and places this discourse in a South African context, encouraging a pragmatic but thoughtful approach.

We hope this series gives you useful information on some aspects of genetics in medicine, as well as a glimpse into the future of genomic medicine in the 21st century. Similar to Thomas Hunt Morgan's realisation, almost a century ago, that advances in understanding of genetics allow us to find nature to be 'entirely approachable', we hope that this introduction to genetics provides you with a platform to better understand the biology of human physiology and function, as well as pathology.

\section{Karen J Fieggen}

Division of Human Genetics, Department of Medicine, Faculty of Health Sciences, Groote Schuur Hospital and University of Cape Town, South Africa

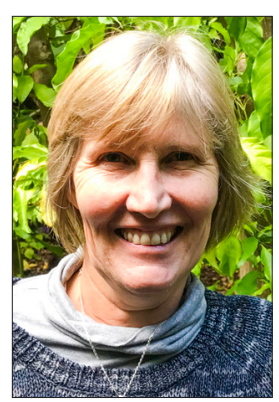

\section{Ntobeko A B Ntusi}

Division of Cardiology, Department of Medicine, Faculty of Health Sciences, University of Cape Town and Groote Schuur Hospital; Cape Universities Body Imaging Centre, Faculty of Health Sciences, University of Cape Town; and Hatter Institute for Cardiovascular Research in Africa, Department of Medicine, Faculty of Health Sciences, University of Cape Town, South Africa

ntobeko.ntusi@uct.ac.za

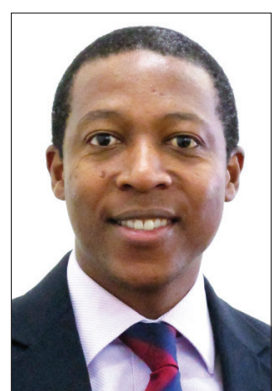

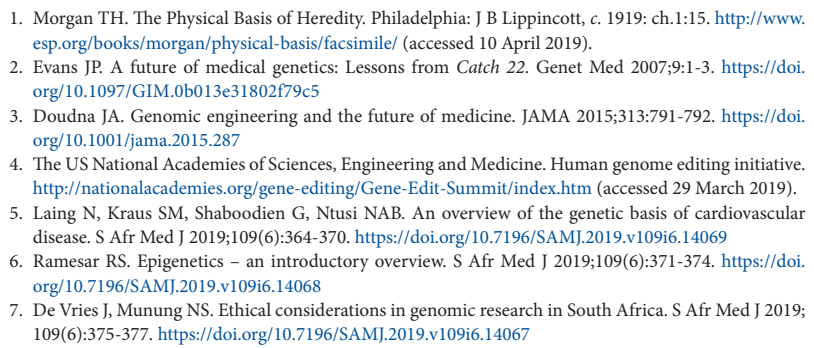

Morgan TH. The Physical Basis of Heredity. Philadelphia: J B Lippincott, c. 1919: ch.1:15. http://www. esp.org/books/morgan/physical-basis/facsimile/ (accessed 10 April 2019).

2. Evans JP. A future of medical genetics: Lessons from Catch 22. Genet Med 2007;9:1-3. https://doi. org/10.1097/GIM.0b013e31802f79c5

3. Doudna JA. Genomic engineering and the future of medicine. JAMA 2015;313:791-792. https://doi. org/10.1001/jama.2015.287

The US National Academies of Sciences, Engineering and Medicine. Human genome editing initiative. http://nationalacademies.org/gene-editing/Gene-Edit-Summit/index.htm (accessed 29 March 2019). . Laing N, Kraus SM, Shaboodien G, Ntusi NAB. An overview of the genetic basis of cardiovascular disease. S Afr Med J 2019;109(6):364-370. https://doi.org/10.7196/SAMJ.2019.v109i6.14069 6. Ramesar RS. Epigenetics - an introductory overview. S Afr Med J 2019;109(6):371-374. https://doi. org/10.7196/SAMJ.2019.v109i6.14068

De Vries J, Munung NS. Ethical considerations in genomic research in South Africa. S Afr Med J 2019; 109(6):375-377. https://doi.org/10.7196/SAMJ.2019.v109i6.14067

S Afr Med J 2019;109(6):362-363. DOI:10.7196/SAMJ.2019.v109i6.14070 